\title{
The HST/ACS Survey of Galactic Globular Clusters
}

\author{
Ata Sarajedini \\ Department of Astronomy, University of Florida, 211 Bryant Space Science Center, \\ Gainesville, FL 32611, USA \\ email: ata@astro.ufl.edu
}

\begin{abstract}
We present an overview and the first results of a large ACS survey of Galactic globular clusters. This Hubble Space Telescope (HST) Treasury project is designed to obtain photometry with $\mathrm{S} / \mathrm{N} \gtrsim 10$ for main sequence stars with masses $\gtrsim 0.2 M_{\odot}$ in a sample of globulars using the Advanced Camera for Surveys (ACS) Wide Field Channel. Our sample consists primarily of globular clusters within about $20 \mathrm{kpc}$ of the Sun with fairly low reddening. We also added a few clusters of special interest toward the Galactic bulge as well as six clusters believed to be members of the Sagittarius dwarf spheroidal galaxy. This sample of 66 globular clusters was observed with HST using 134 orbits. We plan to explore a wide variety of scientific issues with these data including the formation timescale of the Milky Way halo, the age of the Universe, the dynamical evolution of stars in globular clusters (e.g. mass segregation), and the mass of the Milky Way and its gravitational potential field.
\end{abstract}

Keywords. Hertzsprung-Russell diagram, stars: horizontal-branch, stars: Population II, globular clusters: general, globular clusters: individual (NGC 5466, M92, Palomar 1, 47 Tuc), Galaxy: halo

\section{Introduction}

Globular clusters (GCs) are powerful tracers of the Galactic halo, thick disk, and bulge populations. The ages, populations, and kinematics of GCs bear the imprint of the Galaxy's early formation history. Cluster stars - which have a range of masses but a common origin, age and initial chemical composition - play a pivotal role in studies of stellar and cluster dynamics, stellar structure and evolution, and in the formation and evolution of the Galaxy. The only way we can unlock the secrets of the GCs and fully exploit what they have to tell us about our Universe is via the construction of an extensive and uniform database of photometry and astrometry for individual stars in clusters with a range of properties. With these considerations in mind, we are conducting an imaging survey of Galactic GCs using the Advanced Camera for Surveys Wide Field Channel (ACS/WFC) on HST in the F606W and F814W filters.

1) These observations are facilitating a systematic study of both lower main sequence stars, to $\sim 0.2 M_{\odot}$ with $\mathrm{S} / \mathrm{N} \geqslant 10$, and the brightest degenerates $(\geqslant 0.5 \mathrm{mag})$ on the white dwarf (WD) cooling sequence. This ACS GC dataset will be of unprecedented depth and photometric precision and will be used to: a) determine distances via comparison with local subdwarfs; b) measure relative and absolute GC ages; c) obtain the mass function of the central regions, and provide an observational measurement of the mass segregation in the inner regions of GCs.

2) Taking advantage of previous WFPC2 images, we will measure proper motions of the brightest stars in key clusters. This will allow us to: a) separate cluster members from field stars, particularly for contaminated disk/bulge clusters, b) determine proper motion dispersions in order to study their internal dynamics, c) determine distances by 
comparing the proper motion and radial velocity distributions, d) determine absolute proper motions for GCs with respect to background galaxies and hence 3-D velocities and orbits, e) probe the structure and kinematics of the Milky Way, and f) provide a synergy with observations from the Space Interferometry Mission (SIM).

3) Finally, and perhaps most importantly, this dataset provides lasting 'added value' to the HST archive and an enduring legacy of HST, facilitating studies that we have not even considered. By establishing a consistent and uniform deep imaging survey of GCs, HST will enable a host of potential programs.

In this contribution, we present an overview of the project and some of our colormagnitude diagrams (CMDs). More detail on these results is provided in the paper by Sarajedini et al. (2007).

\section{Observations and Data Reduction}

Our target list includes 66 GCs chosen by a number of criteria, the most important of which are proximity to the Sun $\left((m-M)_{0} \leqslant 16.5\right)$ and low-reddening $(E(B-V) \leqslant 0.35)$. However, we have also included a few clusters of intrinsic interest such as those believed to be associated with the Sagittarius dwarf spheroidal galaxy and a number of clusters in the direction of the Galactic bulge. The full details of our target list will be published in a forthcoming paper.

Each cluster in our sample was centered in the ACS field and observed for two orbits, one orbit for each filter, with one short exposure per filter and four to five long exposure frames. The long exposures were dithered to fill in the gap between the two CCDs of ACS. The two exceptions to this observing strategy were Palomar 2 which was not imaged with short exposures and NGC 6715 (M54), which was observed for 4 orbits.

The process of deriving photometry from the short and long exposures and combining the results is briefly described in the first paper on the project by Sarajedini et al. (2007) and will be fully discussed in the upcoming work by Anderson et al. (2007). For the moment, we note that the 'first pass' photometry reported on here and presented in Sarajedini et al. (2007) includes only the uncrowded stars (i.e. those with no brighter neighbors within 3 pixels). The 'final pass' reduction, where we relax this crowding restriction, will include many more stars and will therefore be more complete. The photometric catalog that the Treasury project makes available to the community will include only the final pass data.

We calibrate the photometry to the ACS/WFC VEGAmag system following the procedure given in Bedin et al. (2005) using the most updated encircled energy distributions and the official zeropoints given by Sirianni et al. (2005). The result of this procedure is photometry that reliably extends from the horizontal branch (HB) to several magnitudes below the main sequence turnoff (MSTO).

\section{Results}

The left panel of Fig. 1 shows our color-magnitude diagram (CMD) for the metal-poor globular cluster NGC 5466 in the ground-based VI system. For comparison, the right panel is the CMD for this cluster from one of the most extensive ground-based surveys of Galactic GCs by Rosenberg et al. (2000). The HST CMD is significantly deeper than the ground-based one, and the cluster principal sequences from the HB to the faintest extent of the main sequence are much better defined. In addition, the HST CMD reveals a rich population of unresolved main sequence (MS) binaries as evidenced by the parallel sequence located as much as 0.75 mag brighter than the MS. Note that the scatter among 


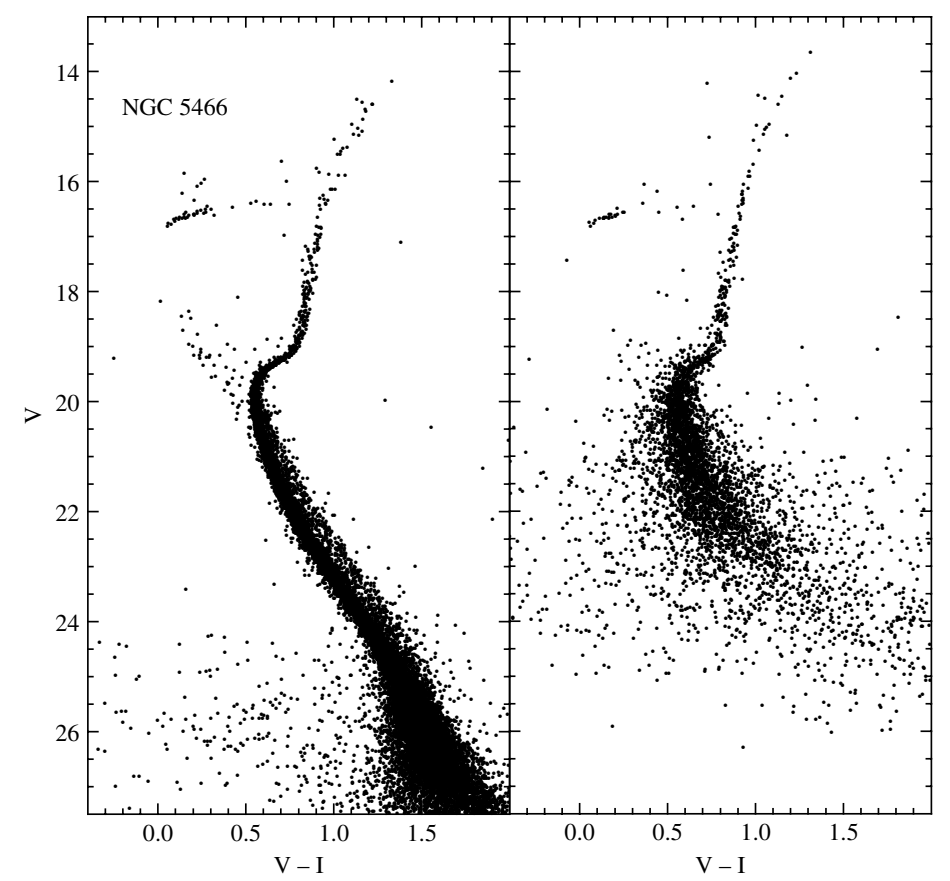

Figure 1. A comparison of our Treasury data (left panel) with ground based photometry for the Galactic globular cluster NGC 5466 from Rosenberg et al. (2000). Not only are the HST/ACS data deeper but they also reveal the cluster principal sequences much better than the ground-based data.

the stars above the HB is larger than it should be for the HST CMD. This is because all of these stars are saturated on our frames, which were designed to optimize the photometry of stars fainter than the HB.

In Fig. 2, we show two examples of MS fitting to comparison clusters of similar metal abundance. The left panel illustrates the NGC 5466 photometry compared with the fiducial sequence of M92 while the right panel compares Palomar 1 with the fiducial of 47 Tuc. The fiducial sequences have been shifted in color and magnitude to optimize the fits to the unevolved MS. Because the main sequence presents a curved shape and is exceedingly well-defined, it is possible to uniquely determine both relative reddenings and distance moduli using this method. These values and their implications are fully discussed in Sarajedini et al. (2007).

\section{Acknowledgements}

I would like to acknowledge the other members of the ACS Treasury team. The core team is composed of (in alphabetical order) Jay Anderson (Rice), Antonio Aparicio (IAC), L. Rolly Bedin (ESO), Brian Chaboyer (Dartmouth), Ivan King (Washington), Steve Majewski (Virginia), Giampaolo Piotto (Padova), I. Neill Reid (STScI), Alfred Rosenberg (IAC), and Mike Siegel (Texas). Since we began the project, Maren Hempel (Florida), Antonio Marín-Franch (Florida and the IAC) and Nathaniel Paust (STScI) have been involved as postdoctoral researchers. Finally, Aaron Dotter (Dartmouth) and 


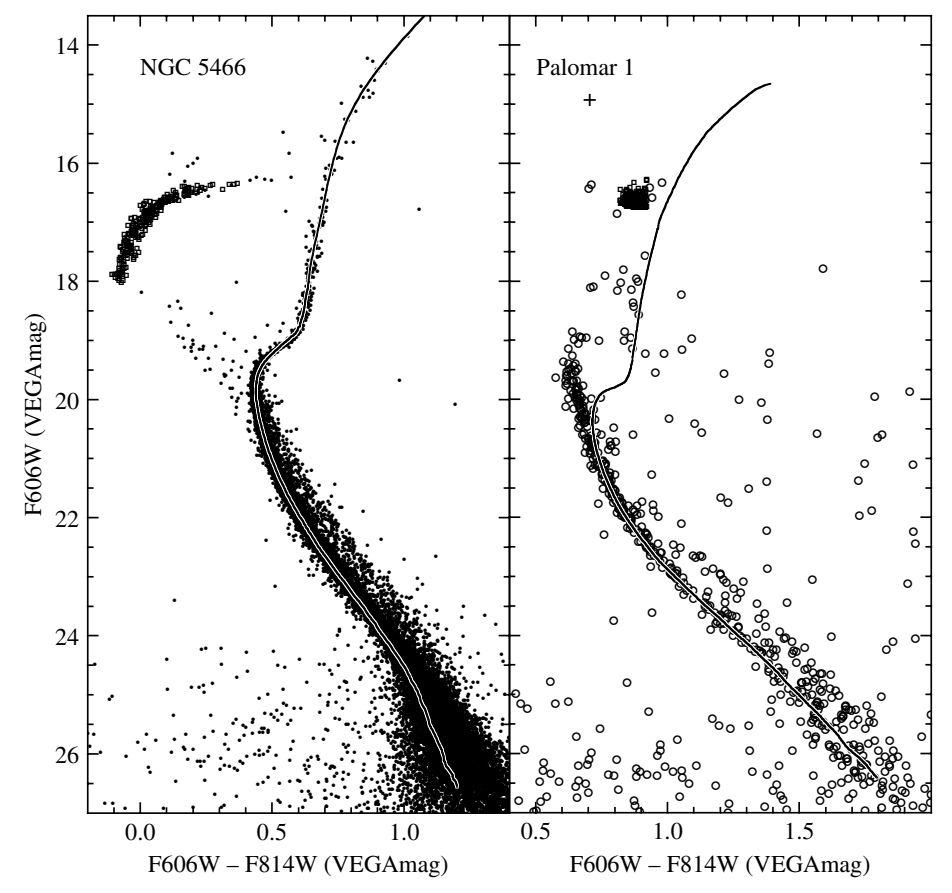

Figure 2. The left panel shows our photometry for NGC 5466 compared with the fiducial sequence (solid line) and horizontal branch (open squares) of M92, also from the Treasury project. These two clusters have similar metallicities so the M92 data have been shifted to match the unevolved main sequence of NGC 5466. The tight correspondence between the data and the fiducial sequence suggests that NGC 5466 and M92 have indistinguishable ages and metallicities. The right panel is similar to the left except that photometry for Palomar 1 is compared with the fiducial and HB of 47 Tuc. The mismatch between the photometry and the fiducial in the region of the turnoff suggests that Pal 1 is significantly younger than 47 Tuc.

Antonino Milone (Padova) have worked on the project as graduate students. Support for this work (proposal number GO-10775) was provided by NASA through a grant from the Space Telescope Science Institute which is operated by the Association of Universities for Research in Astronomy, Incorporated, under NASA contract NAS5-26555.

\section{References}

Anderson, J. et al. 2007, in preparation

Bedin, L., Cassisi, S., Castelli, F., Piotto, G., Anderson, J., Salaris, M., Momany, Y., \& Pietrinferni, A. 2005, MNRAS, 357, 1038

Rosenberg, A., Aparicio, A., Saviane, I., \& Piotto, G. 2000, A\&ApS,145, 451

Sarajedini, A. et al. 2007, $A J$, in press (April)

Sirianni, M. et al. 2005, PASP, 117, 1049

\section{Discussion}

Kroupa: Most of the CMDs had a secondary MS. Are these binaries or blends?. 
SARAJEDINI: These are generally speaking binary stars. Because we are looking at the centers of these clusters, we see rich populations of binaries as a result of mass segregation.

ANDERSEN: Do you see any interesting correlations of the binary population with cluster properties (concentration, etc.).

SARAJEDINI: We are looking into this question. By the time these proceedings are published, it is hoped that we will have published a paper on this topic. The reader is referred to the referred literature for the answer to your question.

PEssev: Do you plan to extend the study in the NIR when WFC3 becomes operational?

SARAJEDINI: Yes, we have discussed this idea among members of the group.

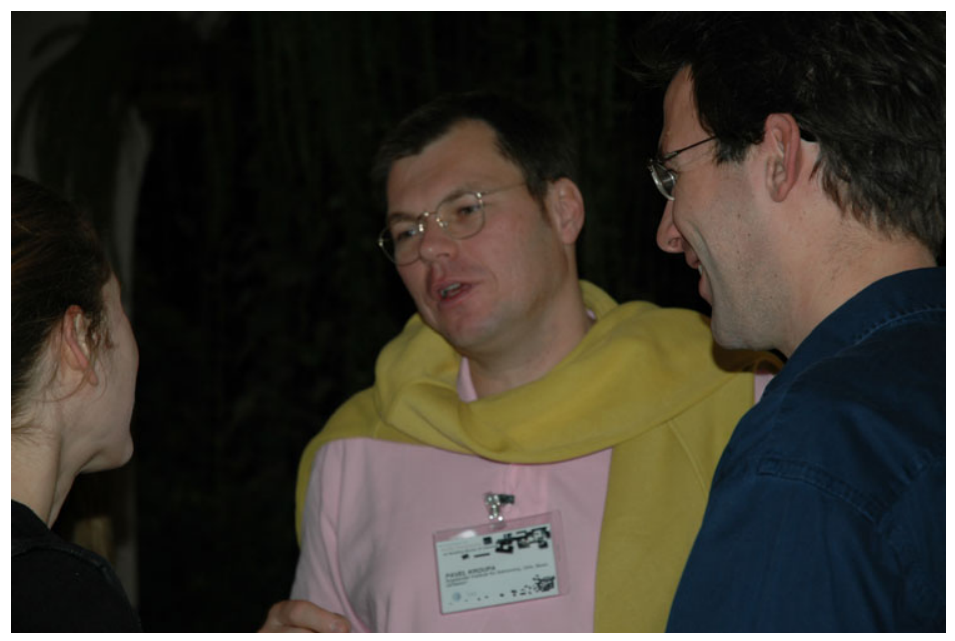

Pavel Kroupa (middle) and Jakob Walcher (right).

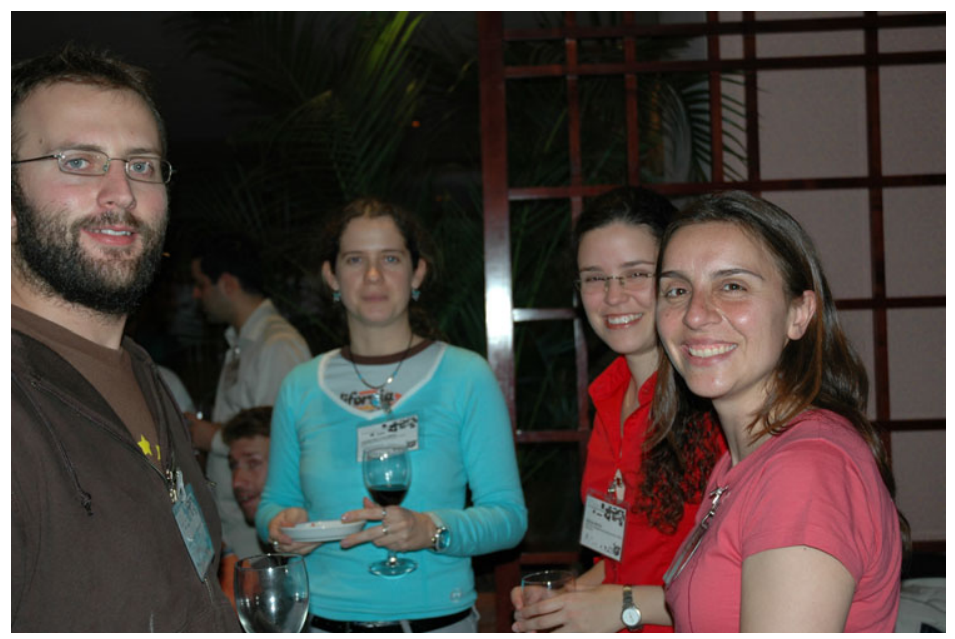

Carlos González-Fernández, Annalisa Calamida, Cecilia Mateu and Sonia Duffau (from left to right). 